Pi. $0^{\text {h251 }}$

Str. Pos. Med. 76

W. $0^{\mathrm{h} 904}$

Moscou Ann. I.

Santini V. 82

Radcl. Obs. 1860-i4

\begin{tabular}{|c|} 
Mean \\
year \\
1800 \\
1824 \\
1825 \\
1861 \\
1863 \\
1869
\end{tabular}

1877.0



\begin{tabular}{|r|c|} 
& $\begin{array}{c}\text { Nr. of. } \\
\text { Obs. }\end{array}$ \\
$7^{\prime} 16^{\prime \prime} 9$ & 4 \\
10.5 & 6 \\
13.1 & 2 \\
5.6 & 5 \\
7.4 & 2 \\
5.2 & 6
\end{tabular}

Besid. errors.

\begin{tabular}{l|l}
-0809 & -1.7 \\
+0.04 & +1.1 \\
-0.33 & -1.6 \\
-0.01 & +0.6 \\
-0.12 & -1.5 \\
+0.10 & -0.2
\end{tabular}

Giving to 1 observation of Piazzi and Weisse only half the weight the position results by the method of least squares $1877.0+t$ :

$$
\begin{aligned}
& x=0^{\mathrm{h}} 53^{\mathrm{m}} \mathrm{j}^{\mathrm{s}} 13-0^{\mathrm{s}} 06 . \mathrm{t} \\
& \delta=0^{\prime \prime} 7^{\prime} 3^{\prime \prime} 8-0^{\prime \prime} \mathrm{i} 48 . \mathrm{t}
\end{aligned}
$$

with the residual errors as shown in the last columns.

Estimates of magnitude of the planet and remarks.

Oct. 14. Sky very fine. The planet is 11 . mag., perlaps even $10^{\mathrm{m}} 5$.

15. Found the planet, but after having adjusted the filar mikrometer, the sky became overcast.

16. Sky fine. Planet $10^{\mathrm{m} 5}$.

28. Very hazy. After 4 comp. with the ringmicr. sky so thick, that planet invisible.

29. Sky tolerably good. Planet $11^{\mathrm{m}}$.

Nov. 7. Sky fine. Planet well seen, 11m.

Dec. 1. Sky not very good, stars blurring, planet estimated $11^{\mathrm{m}} 7$

2. Very fine sky and observations very satisfactory. Planet $11^{\mathrm{m}} 7$.

3. Sky good, Planet well seen $11^{\mathrm{m}} \mathbf{6}$.

8. Interruptions by hazy clouds; between them planet quite conspicuous.

12. Sky with thin hazy clouds, but the planet was seen very well.

25. Fine, after the fog cleared away. The planet appeared quite bright yet; but there was no time for exchanging the ring with the filar micrometer.

26. Sky good; planet well seen.

Jan. 3. Stars much blurring, with the thermometer at $-6^{\prime \prime}$ Fahr. in the morning, and the wind now changing to the south.

6. Stars blurring; observation difficult $12^{\mathrm{m}}$.

9. Clear; south wind; little moonlight. Planet rather faint. about $12 \mathrm{~m}$.

23. Very clear, soon after a snowstorm with therm $+2^{0}$ Fabr. Planet now rather small, perhaps $120^{\circ}$. There is a fine are of bright aurora $20^{\circ}$ high.

24. Strong south wind and stars blurring. Planet difficult.

28. Cold and clear. Planet $12^{\mathrm{m}} 5$.

29. Strong wind, directly upon shutters, so that the lamp would not stand it, and the ringmicrometer lad to be used.

Clinton, N. Y. 1878. May 15.

C. H. F. Peters.

\title{
Bemerkungen über Sternspectra.
}

Ich erlaube mir wieder auf einige Sternspectra aufmerksam zu machen, über welche, wie ich diesmal mit ziemlicher Gewissheit behaupten kann, entweder Nichts bisher veröffentlicht wurde, oder wo meine $\mathrm{Be}$ obachtungen $\mathrm{zu}$ anderen Resultaten als die früheren geführt haben. Die Spectra gehören sämmtlich zur dritten Classe nach Vogel, folglich zur Typ. 1II. oder IV. nach Secchi. Bei den Farbenschätzungen habe ich die Scala von Schmidt (A. N. 1897) angewandt. 
i Virginis $=159 \mathrm{Schj} \quad d=-12^{\circ}$

Secchi sagt (Memoria II), das Spectrum dieses Stems sei rom IV. Typ., und dasselbe wird in seinem „Prodromo di un Catalogo fisico" wiederholt. Meine Beobachtungen an drei Abenden geben aber: Farbo (i.3. Spectrum unzweifelhaft von Cl. III. a mit deutlichen aber recht schwachen Bandeu.

$$
219 \text { Sohj. }
$$$$
\delta=-8^{\circ}
$$

Stern ז. Grüsse, Farbe 8.5. Schünes Spectrum von Cl. III.b, zwar uicht hell, in welchem aber doch die karakteristischen, broiten und nach der violetten Scite verwaschenen Banden in Grín und zwischen Grün und Gelb sebr scharf hervurtreton. Die Banden in Ruth sind dagegen wie gewölınlich zart und nicht ganz leicht sichtbar.

B.D. $+20.2561=$ Var. R. Virginis.

Earbu 5.5. Im Maximo des Sterns war das Spectruin unzweifeliaft ron Cl. III a mit breiten aber schwachen Baudiou.

B.U. : s439s(1)= Var. R. Aquilau.

Farbe i.s. Spectrum rooht sobün von Cl. III. a unit breitrn, duskeln Banden durch allo Farbeu. Die Baulun sind nacl, buiden Beiten verwaschen.

B.J). - $1502 \times 58$.

Fartu i.ll. Deutliolies abor uioht ansgezeichnetes Spectrum rou Cl. III. a.

B.D. $+31^{\circ} 272 \bar{s}=$ Var. S. Curonac.

Farbo 6.2. Schünes Spectrum Cl. III a. Die tiefdunikeln aber diffusen, durch alle Farben sichlbureu Bandeu sind gan\% ungewöhnlich breit. Die belle Colunne in Gelbyrthn wird in Folge dessen fast linienschmal.

B.D. $+35^{\mathrm{v}} 35222=5009$ Birm.

Farbe K.3. Das Spoctrum ist von Cl. III.b, und im Verhältniss zur Lichtstärke liberraschend prachtig. Im Roth und im Orange zwei feine, matte Banden, aber zwischen Gelb und Grun und im Grun zwei breite dunkle Intervalle, wodureh das Spectrum discontinuirlich wird. Indigo und Violett sind unsichtbar, wenigstens bei der grossen Helligkeit der Nuchte zu diesor Jahreszeit.

$$
\text { B.D. }+36^{\circ} 3243=458 \mathrm{Birm} \text {. }
$$

Wie man in Birminghams "Catalogue of the red stars * p. 322 sieht, ist das Spectrum dieses Sterns sclion (von Seochi ?) untersucht worden, und ein Zonenspectrum gefunden; uber die Vertheilung der Banden ist aber Nichts angegeben, folglich Typ. III. gemeint. Meine Beobachtungen geben aber:
Farbe 8.§. Typisches Spectrum ron Cl. III. b mit drei hellen Zonen, Roth, Grim, Blan, getrennt durch zwei breite, nach der violetten Seite verwascheno Baudun.

B.D. $+42^{\circ}: \mathrm{i} 15=" \operatorname{Tirsae} \mathbf{M} \mathrm{aj}$.

Farbe 6.3. Spectrum ron Cl. 1II. a mit scharf geschnittenen. obgleich etwas schwachen Banden durch das gauze Farbenbild.

B.D. $+43^{0} 34 \% \overline{5}=521$ Birm.

Farbe 8 :. Spectrum schwach, aber unzweifelhaft von Cl. III. b. Der ruthgelbe Theil ist von dem grimen durch riue breite Bande retreuut; das Grün bricht auch plïtzlich ab, und erst uacil einom andern breiten Intervilt: \%eigt sish eine schwache Spur rom blauen Lichte Für dio Cutersucitung dieses Sterus sind aber dis: Nichte !ier jetzt viel zu hell.

B.D. $+4.202241=367 \mathrm{~B} \mathrm{irm.}$

Farbe 80 \%. Pracitvolles Spectrum von Cl. III. a. Dic Bunden verlaufen uacb beiden Seiten, sind aber durch alle Farben sehr dunkel und un...wiohnlich breit.

B.I. $+310.24 \pi=320$ Birm.

Farbe 6.5 sehr deutliches Spectrum ron (H. III. a mit reci:t breiten, dunkeln und einseitig scharf begrenzteu ilsorptiuusstruicu.

B.D. $+55^{6} \div 4: 2$.

Farbe $i$ j. Recht !ulisehes Nipectrum Cl. III a, das doch gute: Luft fordert.

B.D. $+5: 0.2521=6 ! 5$ Birm.

Farbe 7. . Ausserordentlich prachtvolles Spectrum Cl. III. a. Die Banden sind so breit, duukel und scharfgeschnition, dass nur wenige Sterne mit so stark ausgepriigten Bandenspectren rorkommen.

B.U. $+60^{\circ} \mathrm{i}+06=\mathrm{Tar} . \mathrm{T}$. Ursae $\mathrm{Maj}$.

Farbe 5.5. Spectrum unzwreifelliaft von Cl. III. a. Die Banden sind zwar unverkenubar durchs ganzeSpectrum, aber relativ ziemlich schwach.

B.D. $+61^{0} 2444$.

Farbe 5.5. Recht hubsches spectrum Ol. III.a. Mit Ausuahme der zwei Banden im Gelbgrun, die ziemlich zart, sind die Streifen breit und leicht sichtbar.

B.D. $+62^{\circ} 2002=599$ Birm.

Farbe 7.5. Spectrum Cl. IlI.a. Die Banden sind gut begrenzt und schön sichtbar durch alle Farben.

B.D. $+6202028=601 \mathrm{~B} \mathrm{i} \mathrm{rm.}$

Farbe 7.0. Prachtrolles spectrum von Cl. HI. a. Die Banden sind breit und tiefdunkel durchweg. 
B.D. $+64^{\circ} 1405$.

Farbe 6.j. Spectrum von Cl. III. a mit Banden, die im Roth und Orange gut sichtbar, im Grin etrras scliwacher sind.

B.D. $+\pi 0 \omega 0 \overline{0}=\alpha$ Draconis.

Farbe 7.j. Recht hubsches Spectrum Cl. III.a mit Banden sichtbar durch alle Farbenzonen.

B.D. +290721 .

Farbe i.jo. Nettes Colonnenspectrum ron Cl. III. a mit breiten. diffusen Banden.

B.D $+\$ \$^{0} 112=i$ Crsae ifin.

Farbe ij.j. Deutliches aber wenig brillantes Spectrum von Cl. III.a.
Schliesslich bemerke iol, dass in meiner frtheren Mittheilung in Nr. 2200 der A. N. durch ein Versehen sich ein Irrthum eingeschlichen hat, indem eins von den Spectren, namlich das von B.D $+66^{\circ} 878$. welches D'Arrest sohon fruber beobachtet hat. als neu aufgefuhrt wurde. In derselben Publication kommt aucls ein Druckfeliler vor. Der Stern $i$ Draconis ist nämlich als B.D. + 650593 bezeichnet. Statt dessen nuss: $+60^{\circ} 963$ gelesen rerden.

Lund den 21. Juni 1878.

\section{A. CIInos.}

Planetenbeubachtungen.

augestullt am le-fussigen Aequatoreal der Leipziger Sternwarte. mitgetheilt rou Herrn Professor C. Brulus.

16. Protogeneia.

\begin{tabular}{|c|c|c|c|c|c|c|c|c|c|c|}
\hline \multirow{2}{*}{ L8T } & \multirow{2}{*}{$\begin{array}{c}\text { Mittl. Zeit } \\
\text { Leipzig }\end{array}$} & \multicolumn{2}{|c|}{ Planet - } & \multirow{2}{*}{ A.R. app. } & \multirow{2}{*}{ Lot. f p. } & \multirow{2}{*}{ Decl. app. } & \multirow{2}{*}{ Lg.f.p } & \multirow{2}{*}{\begin{tabular}{|c|} 
Zahl der \\
Beol.
\end{tabular}} & \multirow{2}{*}{$\begin{array}{l}\text { Tgl. } \\
\text { Stcirs. }\end{array}$} & \multirow{2}{*}{$\begin{array}{c}11 . \\
\text { othactst. }\end{array}$} \\
\hline & & $\because-\infty$ & $J^{\prime}-d$ & & & & & & & \\
\hline an. 10 & $1: 216^{\mathrm{m}} 4$ & 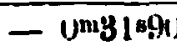 & $+4+10^{\circ} 6$ & $7^{\text {hl }}$ (ym35n-1 & 9.14 iin & $T^{\prime 2} 1^{\prime \prime} \times 3^{\prime}$ & 0.0508 & $12: 4$ & 1 & $\mathrm{p}$ \\
\hline 23 & 9.2 & $-19 . \overline{3} 1$ & +2. & (i.j) 2 s4 & y. (xitisn & +21.1549 & 51 & $1 \times: 13$ & 2 & $\mathbf{P}$ \\
\hline (1) & 111.10 .311 & -213.9 & -4.39 .5 & 6.48 .35 .46 & $8.31+n$ & +21.20 .12 & (5) 643 & $18: \cdot 1$ & $\ddot{3}$ & $\mu$ \\
\hline 28 & T.tii.20 & -11.40 .5 & $-\bar{j} . \overline{3} 3.1$ & $6: 46$ 35.27 & y $379 n$ & +-21.22 .9$. & 0.681 & $18: i i$ & 4 & $\mu$ \\
\hline
\end{tabular}

(3i) Fides.

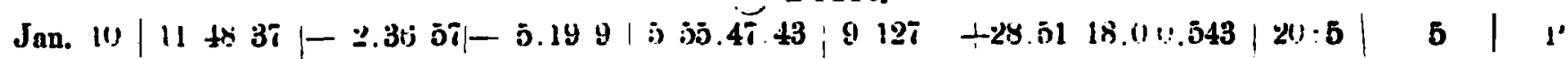

ing Ex.

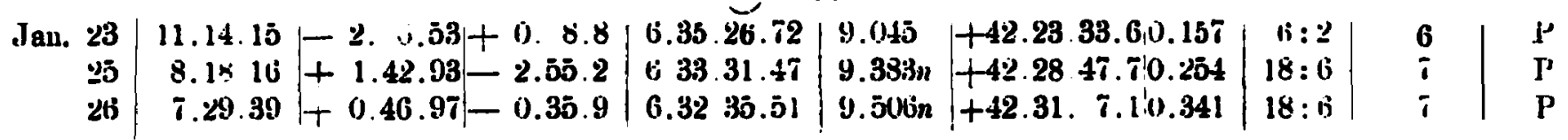

Leto.

\begin{tabular}{r|r|r|r|r|r|r|r|r|r} 
Jan. 25 & 12.4 .51 & +1.31 .25 & +2.0 .3 & 7.8 .9 .40 & 9.164 & +3.11 .57 .20 .461 & $18: 6$ & 8 & $p$ \\
26 & 9.5 .215 & $+0.44 .63 \mid+2.23 .4$ & $\tau .7 .22 .78$ & $9263 n$ & +33.12 .20 .30 .476 & $8: 3$ & 8 & $p$
\end{tabular}

(118) Amalthea.

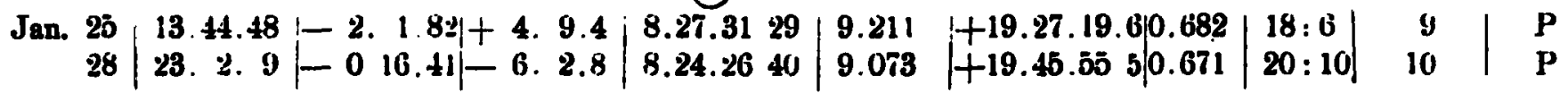

III: Cassandra.

Jan. $28|11.59 .23++0.15 .57|+0.51 .1|7.41 .49 .48| 8.918|+15.31 .5 .20 .714| 24: 12\} 11 \quad P$

(10) Rhodope.

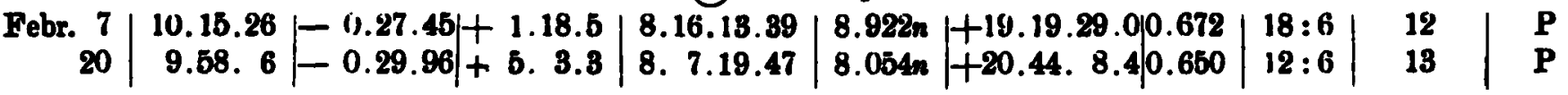

